\title{
Minimal Hepatic Encephalopathy among Cirrhotics A Cross Sectional, Clinico-Epidemiological, Multi-Centre, Study in Patients of Pakistan
}

Arif M Siddiqui ${ }^{1}$, Javed Farooqi ${ }^{2}$, Muhammad B Nasir ${ }^{3}$, Karim Kammeruddin ${ }^{4}$, Ghias N Tayyab ${ }^{5}$, Arif A Nawaz ${ }^{6}$, Masood Siddiq ${ }^{7}$, Junaid Saleem ${ }^{8}$, Sobia Ali ${ }^{9 *}$, Raeefuddin Ahmed ${ }^{10}$

${ }^{1}$ Professor of Medicine, Consultant Gastroenterologist, Pakistan Kidney and Liver Institute, Knowledge City، 1, PKLI Avenue, Opposite DHA Phase 6، Sector E Chota Mota، Singh, Lahore, Pakistan

${ }^{2}$ Professor, Consultant Gastroenterologist, Lady Reading Hospital, Soekarno Rd, PTCL Colony Peshawar, Khyber Pakhtunkhwa 25000, Pakistan

${ }^{3}$ Assistant Professor, Lahore General Hospital, Ferozpur Road، near Chungi، Amar Sidhu Ismail Nagar, Lahore, Punjab 54000, Paki stan

${ }^{4}$ Professor of Medicine, Baqai Medical University and Hospital, 51, Deh Tor, Gadap Road, Near Toll Plaza, Super Highway P.O Box No 2407, Karach - 74600, Pakistan

${ }^{5}$ Professor \& Head of Department, Fatima Memorial Hospital, Shadman Rd, Ichhra Lahore, Punjab 54000, Pakistan

${ }^{6}$ Consultant Gastroenterologist, Lahore General Hospital, Ferozpur Road، near Chungi، Amar Sidhu Ismail Nagar, Lahore, Punjab 5 4000, Pakistan

${ }^{7}$ Consultant Gastroenterologist, Yusra Medical College, Masood Siddiq, Talha Road، Zaraj Housing Society Sector B Isl amabad, Islamabad Capital

Territory

${ }^{8}$ Consultant Gastroenterologist, Hearts International Hospital, Unnamed Road, Saddar, Rawalpindi, Punjab 46000, Pakistan

${ }^{9}$ Senior Medical Manager, Abbott Laboratories, Abbott Laboratories, 8th Floor, Faysal House, Shahrah e Faisal, ST 02, Karachi, Pakistan

${ }^{10}$ Medical Director, Abbott Laboratories, Abbott Laboratories, 8th Floor, Faysal House, Shahrah e Faisal, ST 02, Karachi, Pakistan

DOI: $10.36348 /$ simps.2019.v05i11.001

| Received: 16.10.2019 | Accepted: 25.10.2019| Published: 06.11.2019

*Corresponding author: Sobia Ali

\section{Abstract}

This was a prospective, multicenter, observational and non-interventional epidemiological study. Five hundred and fifty (550) male and female patients between 18 to 65 years with an established liver cirrhosis were enrolled. Patients recruited underwent screening of mental state by Mini Mental State Examination (MMSE). Patients with a score of MMSE $\geq 24$ were required to undergo Psychometric Hepatic Encephalopathy Score (PHES) examination to diagnose MHE. Subjects with an abnormal PHES score of -5 or less were diagnosed with MHE. Subjects with established MHE and those without MHE were assessed for quality of life (QoL). QoL was assessed for Health-Related Quality of Life (HRQoL) using the SF-36 questionnaire. The proportions for the physical component scores (PCS) and mental component scores (MCS) of the SF-36 were calculated and compared. Among the diagnostic scores the mean MMSE score was $27.5 \pm 1.8$. Of these subjects, 451 were found to have a MMSE score of 24 or higher and were administered the PHES examination. Two hundred and fifty-six (256) subjects $(56.8 \%$ ) had an abnormal score of $\leq-5$ and were diagnosed as having minimal hepatic encephalopathy (MHE). Subjects were tested for quality of life through SF-36 questionnaire. Subjects with established MHE were found to have a significantly lower physical component score (PCS) compared to those without MHE. Frequency of Minimal Hepatic Encephalopathy was found to be $56.8 \%$ of patients with Chronic Liver Disease presenting to clinics. Impact of MHE on physical component of quality of life may be of clinical relevance.

Keywords: Minimal Hepatic Encephalopathy, cirrhosis, chronic liver disease.

Copyright @ 2019: This is an open-access article distributed under the terms of the Creative Commons Attribution license which permits unrestricted use, distribution, and reproduction in any medium for non-commercial use (NonCommercial, or CC-BY-NC) provided the original author and source are credited.

\section{INTRODUCTION}

Cirrhosis is the end result of injury to the liver due to various insults leading to fibrosis and nodular regeneration. It is one of the most common causes of morbidity and mortality both globally and in our country [1-3]. In a developing country like Pakistan where cost of health care has always been an issue, chronic diseases like cirrhosis and its various complications are a large burden on health economy. Inadequate education, poverty, poor hygienic environment, inadequate nutrition and lack of counselling are important factors due to which the number of cirrhotic patients is increasing and is causing increased mortality in these patients due to various complications [1].

Hepatic encephalopathy (HE) is a potentially reversible, metabolically caused disturbance of the central nervous system function that occurs in patients with acute or chronic liver disease. It encompasses a broad spectrum of neurological symptoms of varying severity [4]. The stages of overt encephalopathy are (1) mild confusion, (2) drowsiness, (3) stupor, and (4) coma. Revised staging system encompasses absent, 
covert and stages 2 to 4 encephalopathy. Minimal hepatic encephalopathy (MHE), previously known as subclinical or latent hepatic encephalopathy is the mildest form of $\mathrm{HE}$, that is included in covert encephalopathy and is characterized by subtle motor and cognitive deficits with impairment of health-related quality of life (HRQOL) [5-7]. Patients with MHE have impairment in attention, visuospatial perception, speed of information processing, especially in the psychomotor area, fine motor skills, and short-term memory [8].

Liver cirrhosis is a common cause of mortality amongst the Pakistani population and a frequent cause of admission in our hospitals [2] and the majority of patients with chronic liver disease have evidence of hepatitis B (22\%), hepatitis C (28\%) viral infection ${ }^{3}$

Liver cirrhosis causes significant morbidity and mortality, mainly due to complications like hepatic encephalopathy, ascites, hepato-renal syndrome (HRS) and esophageal variceal hemorrhage $(\mathrm{EVH})$ [3]. Hepatic encephalopathy (HE) is present in up to $70 \%$ of all patients with cirrhosis, including patients with abnormalities demonstrable only by psychometric testing [9, 10], out of which about $30 \%$ patients of chronic liver disease die of hepatic encephalopathy [1113].

There is no recognized gold standard test for the diagnosis of MHE, so different neuropsychological tests along with varied cutoffs are used in a varied population of patients depending on the study or center involved.

Cirrhotic patients with MHE more frequently develop episodes of overt HE than those without MHE $[7,15]$. In one study the probability of overt $\mathrm{HE}$ at 3 years was $56 \%$ for those with MHE and $8 \%$ for those without $\mathrm{MHE}^{15}$. It is probable that MHE is a marker of advanced liver failure, for this reason, MHE has been proposed as an indication for liver transplantation [16].

Chronic liver disease is a worldwide major problem and alcohol is the leading cause of chronic liver disease in western societies, while hepatitis B and $\mathrm{C}$ viruses are the major underlying causes in Pakistan. The prevalence of hepatitis B is over $10 \%$ in the Asiapacific region where as the prevalence of chronic hepatitis $\mathrm{C}$ in the Asia-pacific region varies between 4 to $12 \%[17,18]$.

Pakistan is in the intermediate zone of hepatitis B and C prevalence areas. In 2003 and 2004, the prevalence was described as around 4\% (B) and 6\% (C) respectively [19, 20]. In 2010, the Pakistan Medical and Research Council conducted a community-based study showing a reduction in the prevalence rate of hepatitis $\mathrm{B} \& \mathrm{C}$ to $2.5 \%$ and $4.9 \%$ respectively, in the general population [21]. Higher prevalence however, has been reported in certain high risk groups and in family members of hepatitis $\mathrm{C}$ patients.

Hepatic encephalopathy (HE) constitutes one of the principal markers of survival among patients with cirrhosis. As hepatic reserve declines, the probability of acute $\mathrm{HE}$ episodes increases, thus reducing survival [22, 23]. Although it is part of the spectrum of HE, minimal hepatic encephalopathy (MHE) does not manifest clinically and remains underdiagnosed. The prevalence of MHE has been estimated to be between 22 and $80 \%$ [16, 24-27]. This wide range reflects the lack of a gold standard test or absence of standardized diagnostic criteria for MHE across the globe [28-32].

Minimal hepatic encephalopathy negatively affects a patient's health-related quality of life (HRQoL). Activities requiring attention, motor skills, and visual-spatial abilities (i.e., driving a car) are impaired, whereas basic activities of daily living (i.e., eating, bathing, and personal hygiene) are preserved [33].

Using a simple battery of neuro-psychometric diagnostic tests, functional abnormalities associated with MHE can be recognized even before the appearance of overt symptoms. Such an early diagnosis may improve the quality of life and the prognosis of these patients. An epidemiological (non-interventional), multi-center, cross-sectional study was proposed to identify MHE patients from a cohort of stable cirrhotics who do not have any overt symptoms and assess their health-related quality of life using the SF-36 questionnaire. This study contrasts with other prior studies within single centers aiming at measuring the overall burden of MHE in Pakistan. The findings of the study aim at assisting physicians in the evaluation and regular detection of patients potentially affected by MHE in their routine clinical practice. In addition, the study may assist in the design of future studies and for developing a consensus on the clinical management of MHE in Pakistan.

\section{MATERIALS AND METHODS}

This study was conducted respecting the principles of Good Clinical Practice (GCP) Guidelines, and all relevant national guidelines. All information generated in this clinical investigation was considered confidential and was not disclosed to any person not directly concerned with the study without prior written permission from the sponsor.

Patient's written authorization to use and/or disclose personal and/or health data were obtained prior to enrolling each patient in the program and patients not willing to provide such written authorization were not included in the program. All reasonable effort was made to avoid collection of subject identifying 
information (such as name, address, etc.). The physician was responsible for giving the study patient full and adequate verbal and written information about the nature, purpose, possible risks and benefits of the study. The physician was responsible for obtaining signed patient authorization from all patients before including the patient in the observational program.

The protocol and relevant study documents were submitted for review and approval of Institutional Review Board.

Institutional Review Board/ Ethic Committee approval was given by Dr. Ziauddin Hospital IRB located in Karachi, Pakistan dated 23-May-2016. Patient enrollment was started only after obtaining written approval from the IEC.

This was a prospective, multicenter, observational and non-interventional study that recruited patients at eight centers from five major cities of Pakistan between May 2016 and March 2017.

All patients visiting the outpatient department of gastroenterology, hepatology, family medicine or internal medicine for any reason and who were known cirrhotics based on either clinical or biochemical methods or radiological studies or liver biopsy were considered for enrollment.

Liver cirrhosis was considered to be the case if Fibrosis -4 (Fib-4) score was greater than 3.25 [33]. Additionally, any of the data that gave further weightage to the diagnosis which included, patient's history of drug use, blood transfusion, or alcohol use and fatigue due to hepatitis, physical examination revealing Jaundice/ Ascites/ Spider angioma, hepatomegaly and or Splenomegaly were taken into account and documented. Ultrasonography findings of coarse echo pattern of liver by fibrosis and regeneration were also documented.

Inclusion criteria included patients of either sex, 18-65 years of age, having attended school at least till the 5th Class/Standard and having an established diagnosis of liver cirrhosis without any overt symptoms at time of testing with Grade 0 of West Haven criteria. Patients willing to undergo the neuropsychological (NP) tests and to complete the SF-36 questionnaire for the estimation of HRQOL and patients willing to provide written authorization to provide data for the study were subsequently included.
Patients with inability to perform neuropsychometric tests and to complete the SF-36 questionnaire as decided by the physician were excluded from the study.

All patients fulfilling the inclusion criteria underwent screening of mental state by using the MMSE. MMSE consists of eleven questions which measures five areas of cognitive function namely orientation, registration, attention and calculation, recall and language. Around 5 to 10 minutes were taken to administer it.

Patients having a score of MMSE greater than or equal to 24 were subsequently required to undergo the PHES examination to diagnose MHE.

SF-36 Questionnaire was completed by interviewing all patients (with or without $\mathrm{MHE}$ ) for HRQoL evaluation.

PHES consist of a standardized battery of paper-pencil test like number connection tests-A (NCTA), figure connection test $-\mathrm{A}$ (FCT-A), the line-tracing test for time (t) and error (e), the serial-dotting test, and the digit symbol test.

The sum of the error points in each of the PHES examination exercise was to be recorded on a scoring sheet and the raw score converted to a standard score using the appropriate normative data. The conversion for the raw scores to standard scores was based on the following procedure. The performance of a control group $(n=150)$, aged 15 to 80 years, served as the basis for establishing normative values. All scores which were lying within one standard deviation for this group's performance were assigned a score of 0 . Test scores below one, two or three standard deviations were assigned scores of $-1,-2$, or -3 points. Scores higher than one standard deviation were given a score of +1 point. The sum of the standardized scores (summed points) corresponds to the total PHES score, with the possibility to obtain values between +6 and -18 points. Scores below were considered pathological or abnormal.

The Quality of life was assessed by HRQoL using the SF-36 questionnaire. The proportions of lower score and higher score were calculated. The proportions for the physical scores (PCS) and mental scores (MCS) of the SF-36 were calculated and compared between the subjects with MHE and those without MHE. Study design and conduct is shown in Fig-1. 


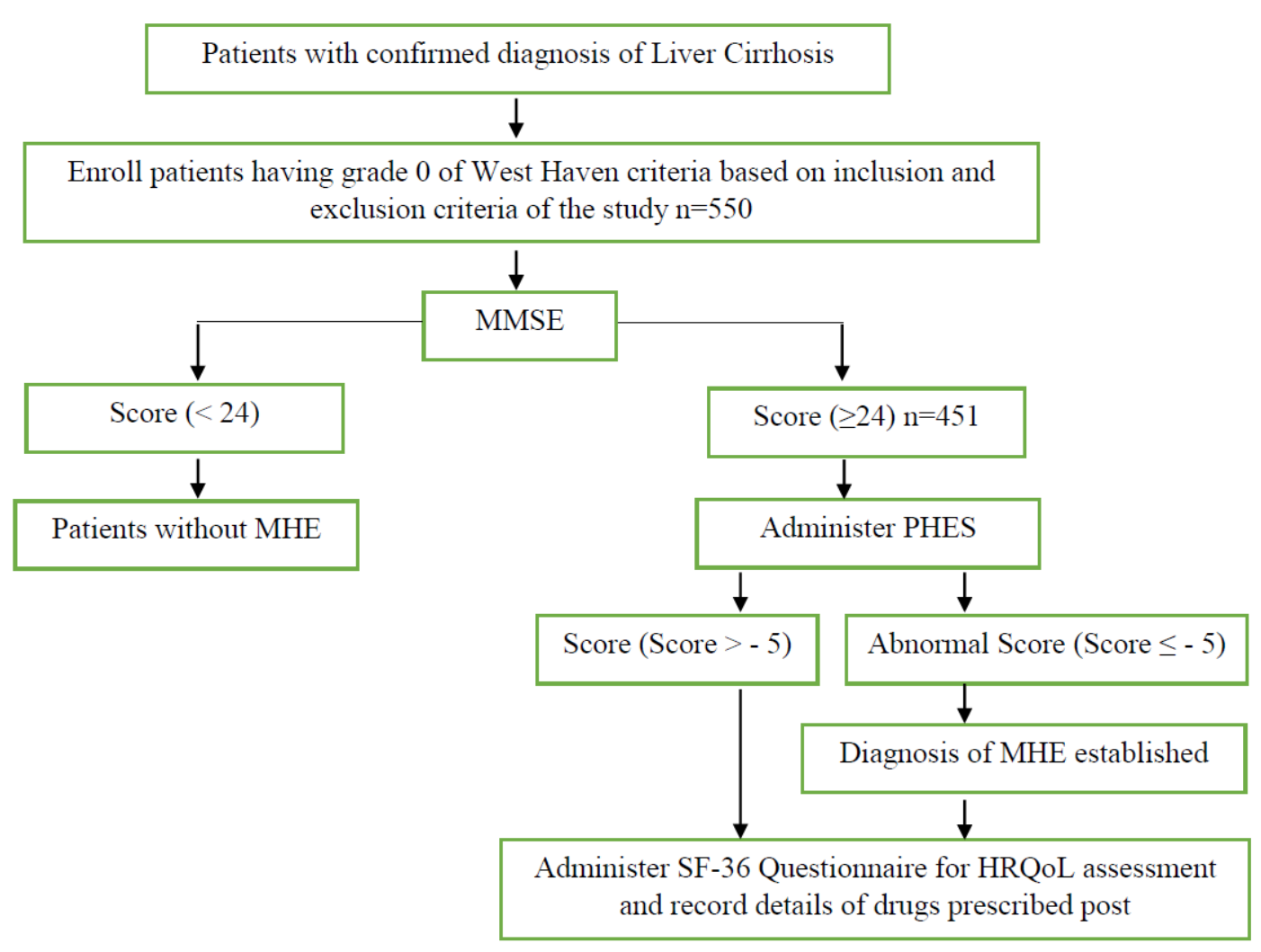

Fig-1: Study Schematic

\section{STATISTICAL ANALYSIS}

All calculations were performed by section of statistics, department of Medicine at The Aga Khan University.

To estimate the prevalence of MHE among cirrhotic patients, using $48.5 \%$ prevalence as reported by an Indian study [40], it was calculated that a maximum sample size of 381 would be required, with $95 \%$ level of confidence and $5 \%(0.05)$ bound on the error of estimation.

To identify the factors associated with MHE, predisposing factors proportion from 29-53\%. Taking into account $95 \%$ confidence interval, $80 \%$ power and odd ratio of 2:00, final sample size will be approximately 289 patients.

To evaluate the HRQoL in MHE patients using the SF-36 questionnaire, assuming a standard deviation of 20 and mean difference of 5 or more points between the two groups is clinically and practically relevant, given a standardized effect size of 0.25 , using this standardized effect size with a two-sided 5\% significance level and $80 \%$ power generated the estimated required number of subjects per group as 253 MHE patients $(n=500)$.

It was concluded that a sample size of 500 cirrhotic patients would achieve all the study objectives.
The sample size was further inflated by $10 \%$ up to 550 to account for drop outs.

Continuous variables with normal and nonnormal distributions has been reported as mean (SD) and median [inter-quartile range (IQR)], respectively. Prevalence of MHE in cirrhotic patients with $95 \%$ confidence Interval (CI) has been calculated. Continuous variables between group compared by using Independent sample $\mathrm{t}$ test, and categorical variables compared by using the chi-square test. The likelihood ratio test used to assess the association between the explanatory variables and the risk of the MHE. Univariable analyses will perform to examine the effect of each variable on the risk of MHE. In multivariate analysis all insignificant variables $(p>0.05)$ will check for their confounding and interaction effects before their removal from the final model. The Quality of life will be assessed by HRQoL using the SF-36 questionnaire. The proportions of lower score and higher score will be calculated. The proportions for the physical scores (PCS) and mental scores (MCS) of the SF-36 will be calculated. Intergroup comparisons would be conducted using the Mann-Whitney U-test. Correlation analyses will perform using Spearman's rank correlation analysis.

All p-values will base on two-sided tests and significance will be set at a p-value less than 0.05 . The 
analyses will perform using SPSS (Statistical Package of Social Sciences) version 19.

\section{RESULTS}

A total of five hundred and fifty participants were recruited in this study (Table-1). The mean age was $47.8( \pm 8.9)$ years (range 22-65 years). There was a preponderance of males $306(68.0 \%)$ versus females $144(32.0 \%)$. The majority of the participants were married 418 (92.9\%), $312(69.3 \%)$ participants had primary schooling.

Out of the subjects enrolled, 451 had MMSE score of $\geq 24$ and underwent PHES testing. Out of the subjects that underwent PHES evaluation, 256 had an abnormal score of $\leq-5$ and were diagnosed as having MHE.

Table-1: Descriptive Characteristics of Study Population $n=451$

\begin{tabular}{|l|l|}
\hline Characteristics & $\mathbf{n}(\boldsymbol{\%})$ \\
\hline Age, in years; mean $\pm \mathrm{SD} ;$ Median[IQR] & $48 \pm 8.9 ; 49[42-55]$ years \\
\hline$\leq 40$ & $98(21.7)$ \\
\hline $41-50$ & $183(40.6)$ \\
\hline $51-60$ & $143(31.7)$ \\
\hline$>60$ years & $27(6.0)$ \\
\hline Gender & \\
\hline Male & $306(67.8)$ \\
\hline Female & $145(32.2)$ \\
\hline Height $(\mathrm{cm}) ;$ mean \pm SD; Median[IQR] & $165.6 \pm 9.4 ; 165.6[157.4-172.7]$ \\
\hline Weight $(\mathrm{kg}) ;$ mean $\pm \mathrm{SD} ;$ Median[IQR] & $68.1 \pm 11.8 ; 67[60-74]$ \\
\hline BMI; mean \pm SD; Median[IQR] & $24.9 \pm 4.2 ; 24.5[22.1-26.8]$ \\
\hline$<18.5$ Underweight & $16(3.5)$ \\
\hline $18.5-24.9$ Normal & $240(53.2)$ \\
\hline $25-29.9$ Overweight & $152(33.7)$ \\
\hline$\geq 30$ Obese & $43(9.5)$ \\
\hline Marital status & \\
\hline Married & $419(92.9)$ \\
\hline Single & $24(5.3)$ \\
\hline Divorced/separated & $8(1.8)$ \\
\hline Education & \\
\hline Schooling & $312(69.2)$ \\
\hline Intermediate & $80(17.7)$ \\
\hline Graduate and above & $59(13.1)$ \\
\hline & \\
\hline
\end{tabular}

Overall the MHE rate was $56.8 \%$ (95\% CI, 52$61)$. MHE was found predominantly in men $(66 \%)$.

The most common etiological cause for cirrhosis was hepatitis $\mathrm{C}$ followed by hepatitis $\mathrm{B}$ in the group with established MHE. In the non-MHE group, hepatitis B was the most common cause of cirrhosis, followed by hepatitis $\mathrm{C}$ (Table-2).

Table-2: Etiology of Cirrhosis

\begin{tabular}{|l|l|l|}
\hline Etiology & MHE n=256 & Non-MHE (n=195) \\
\hline Hepatitis B & $62(24.2 \%)$ & $103(52.8 \%)$ \\
\hline Hepatitis C & $172(67.2 \%)$ & $87(44.6 \%)$ \\
\hline Hepatitis B + C & $13(5 \%)$ & $2(1 \%)$ \\
\hline Alcoholic cirrhosis & $3(1.2 \%)$ & $1(0.5 \%)$ \\
\hline Autoimmune hepatitis & $3(1.2 \%)$ & - \\
\hline Wilson's disease & $1(0.4 \%)$ & - \\
\hline Unknown cause & $2(0.8 \%)$ & $2(1 \%)$ \\
\hline
\end{tabular}

Quality of Life (QoL) was evaluated for all 451 subjects by administrating the SF-36 questionnaire. QoL was compared between the group with MHE $(n=256)$ and the non-MHE group $(n=195)$.
The Mental Component Score (MCS) and Physical Component Score (PCS) were calculated for both groups and were compared.

The average MCS score in the MHE group was 38.21 compared to 39.6 in the non-MHE group. Regarding PCS, there was a statistically significantly 

non-MHE group (44.89) (Table-3).

Table-3: Comparison of factors among MHE and non-MHE patients

\begin{tabular}{|l|l|l|l|}
\hline PCS & $41.8 \pm 7.3$ & $44.8 \pm 6.6$ & $<0.001$ \\
\hline MCS & $38.2 \pm 10.4$ & $39.5 \pm 10.9$ & 0.21 \\
\hline
\end{tabular}

The score for Physical activity level was less in the MHE group. Boxplots of value of MCS and PCS according to MHE status are depicted in Figure-2.

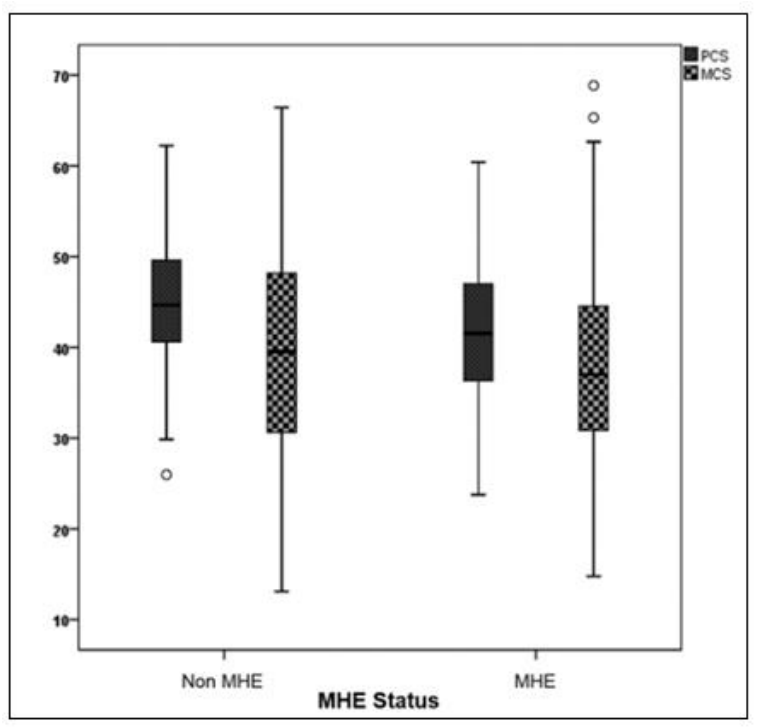

Fig-2: Box plot: Value of MCS and PCS according to MHE status; Boxes represent the 25th, 50th (median) and 75th percentiles

\section{DISCUSSION}

Covert hepatic encephalopathy is prevalent amongst cirrhotics. It does not manifest clinically and requires targeted special testing and therefore there is a high likelihood of being overlooked. The study has demonstrated that patients with MHE have poor quality of life compared to those who do not have MHE, especially where physical impact is concerned.

Various studies have found MHE to be associated with poorer quality of life and increased work disability $[6,34]$.

MHE may increase the risk of accidents. MHE affects attention, psychomotor function and working memory, all of which are essential for safe driving. Most studies assessing driving ability using on-road driving tests have demonstrated that MHE patients have significant defects in reaction time, resulting in their pronouncement as unsafe drivers [35, 37].

Cirrhotic patients with MHE more frequently develop episodes of overt HE than those without MHE [7, 14]. In one study the probability of overt $\mathrm{HE}$ at 3 years was $56 \%$ for those with MHE and $8 \%$ for those without $\mathrm{MHE}^{14}$. It is probable that MHE is a marker of advanced liver failure, for this reason, MHE has been proposed as an indication for liver transplantation [15].
Hepatic encephalopathy (HE) constitutes one of the principal markers of survival among patients with cirrhosis. As hepatic reserve declines, the probability of acute $\mathrm{HE}$ episodes increases, thus reducing survival [21, 22]. Although it is part of the spectrum of HE, minimal hepatic encephalopathy (MHE) does not manifest clinically and remains under diagnosed.

Some studies have identified MHE as an independent predictor of survival in patients with liver cirrhosis. Randomized controlled trials have also indicated that treating MHE leads to an improvement in cognitive abilities and driving performance.

Covert HE includes both MHE and grade I Hepatic Encephalopathy. Although there are no definite guidelines to treat covert $\mathrm{HE}$, various studies have shown efficacy of different drugs. One of the study has shown lactulose therapy to be effective in improving cognitive function and health-related quality of life (HRQOL) in patients of cirrhosis with MHE after 3 months of therapy [6]. In another study, rifaximin use as compared with placebo showed improvement in cognitive function and HRQOL in patients of cirrhosis with MHE [38]. In chronic persistent subclinical HE, LOLA has shown to improve the psychometric test performance and reduce the blood ammonia levels as compared with placebo in individual studies [39]; more data are needed for its use.

\section{LIMITATION OF THE STUDY}

The study was carried out across five cities. In Pakistan, there are towns where the prevalence of chronic liver disease is much higher than other regions. Another limitation of the study was that, although there is international consensus that psychometric tests are the gold standard for the diagnosis of MHE, no agreement exists as to what combination of tests should be carried out and what the threshold value is at which MHE may be reliably diagnosed. This central problem is reflected in the varying reported prevalence for the disease, which range from $22 \%$ to $80 \%$ depending on which test is chosen and definition of the threshold.

\section{CONCLUSION}

The study shows high prevalence of MHE and hence clinicians should consider treating MHE in patients with known cirrhosis. Frequency of Minimal Hepatic Encephalopathy was found to be $56.8 \%$ of patients with Chronic Liver Disease presenting to clinics. Physical Component Score (PCS) of SF-36 was statistically different in patients with MHE compared to 
non- MHE. Impact of MHE on physical component of quality of life may be of clinical relevance.

\section{ACKNOWLEDGEMENTS}

The permission to use the copyrighted questionnaire for the study and training related purposes was obtained from Dr. RadhaKrishan Dhiman of Postgraduate Institute of Medical Education and Research, Chandigarh, India. We are thankful for his and his team's support regarding the above.

The authors are thankful to Abbott Laboratories for providing the research grant for the study in Pakistan.

\section{REFERENCES}

1. Khan, A. U., Iqbal, M., Yasmeen, R., \& Ahmed, W. (2011). Determination of Various Precipitating Factors of Hepatic Encephalopathy in Patients of Chronic Liver Disease at Military Hospital Rawalpindi. Pakistan Armed Forces Medical Journal, 61(4), 626-9.

2. Devrajani, B. R., Shah, S. Z. A., Devrajani, T., \& Kumar, D. (2009). Precipitating factors of hepatic encephalopathy at a tertiary care hospital Jamshoro, Hyderabad. JPMA. The Journal of the Pakistan Medical Association, 59(10), 683-686.

3. Masood, N. (2010). Precipitating factors of Hepatic Encephalopathy in with cirrhosis of Liver. Medical Channel, 16(3); 376-379.

4. Zhan, T., \& Stremmel, W. (2012). The diagnosis and treatment of minimal hepatic encephalopathy. Deutsches Ärzteblatt International, 109(10), 180-187.

5. Ferenci, P., Lockwood, A., Mullen, K., Tarter, R., Weissenborn, K., \& Blei, A. T. (2002). Hepatic encephalopathy-definition, nomenclature, diagnosis, and quantification: final report of the working party at the 11th World Congresses of Gastroenterology, Vienna, 1998. Hepatology, 35(3), 716-721.

6. Prasad, S., Dhiman, R. K., Duseja, A., Chawla, Y. K., Sharma, A., \& Agarwal, R. (2007). Lactulose improves cognitive functions and health-related quality of life in patients with cirrhosis who have minimal encephalopathy. Hepatology, 45(3), 549-559.

7. Das, A., Dhiman, R. K., Saraswat, V. A., Verma, M., \& Naik, S. R. (2001). Prevalence and natural history of subclinical hepatic encephalopathy in cirrhosis. Journal of gastroenterology and hepatology, 16(5), 531-535.

8. Weissenborn, K., Ennen, J. C., Schomerus, H., Rückert, N., \& Hecker, H. (2001). Neuropsychological characterization of hepatic encephalopathy. Journal of hepatology,34(5), 768-773.

9. Gilberstadt, S. J., Gilberstadt, H., Zieve, L., Buegel, B., Collier, R. O., \& McClain, C. J.
(1980). Psychomotor performance defects in cirrhotic patients without overt encephalopathy. Archives of Internal Medicine, 140(4), 519-521.

10. Gitlin, N., Lewis, D. C., \& Hinkley, L. (1986). The diagnosis and prevalence of subclinical hepatic encephalopathy in apparently healthy, ambulant, non-shunted patients with cirrhosis. Journal of hepatology, 3(1), 75-82.

11. Mahboob, F. (2003). Frequency of risk factors for hepatic encephalopathy in patients of chronic liver disease. Annals of King Edward Medical University, 9(1):29-33.

12. Alam, I., Razaullah, H. I., Humayun, M., Taqweem, M. A., \& Nisar, M. (2005). Spectrum of precipitating factors of hepatic encephalopathy in liver cirrhosis. Pak J Med Res, 44(2), 96-100.

13. Nadeem, M., Yousaf, M. A., Zakaria, M., Hussain, T., \& Ali, N. (2005). The value of clinical signs in diagnosis of cirrhosis. Pak J Med Sci, 21(2), 121124.

14. Poordad, F. F. (2007). Review article: the burden of hepatic encephalopathy. Aliment. Pharmacol. Ther; 25(Suppl 1):3-9.

15. Sharma, P. (2009). Minimal hepatic encephalopathy. Journal Assoc Physicians India, 57: 760-763

16. Almani, S. A., Memon, A. S., Memon, A. I., Shah, I., Rahpoto, Q., \& Solangi, R. (2008). Cirrhosis of liver: Etiological factors, complications and prognosis. J Liaquat Uni Med Health Sci, 7(2), 616.

17. Chen, D. S. (2000). Public health measures to control hepatitis $\mathrm{B}$ virus infection in the developing countries of the Asia-Pacific region.Journal of gastroenterology and hepatology, 15, E7-E10.

18. Abbas, Z., Jafri, W., Shah, S. H. A., Khokhar, N., \& Zuberi, S. J. (2004). PGS Consensus statement on management of Hepatitis B virus infection2003. Journal-Pakistan Medical Association, 54(3), 150-158.

19. Hamid, S., Umar, M., Alam, A., Siddiqui, A., Qureshi, H., \& Butt, J. (2004). PSG consensus statement on management of hepatitis $\mathrm{C}$ virus infection--2003. JPMA. The Journal of the Pakistan Medical Association, 54(3), 146-150.

20. Pakistan Medical and Research Council. (2010). National survey on prevalence of hepatitis B \& C in general population of Pakistan. www pmrc org pk/ hepatitisbc html 2010.

21. D‘Amico, G., Garcia-Tsao, G., \& Pagliaro, L. (2006). Natural history and prognostic indicators of survival in cirrhosis: a systematic review of 118 studies. Journal Hepatol, 44: 217-31

22. Stewart, C. A., Malinchoc, M., Kim, W. R., \& Kamath, P. S. (2007). Hepatic encephalopathy as a predictor of survival in patients with end-stage 
liver disease. Liver transplantation, 13(10), 13661371.

23. Dhiman, R. K., Saraswat, V. A., Sharma, B. K., Sarin, S. K., Chawla, Y. K., Butterworth, R., ... \& Madan, K. (2010). Minimal hepatic encephalopathy: consensus statement of a working party of the Indian National Association for Study of the Liver. Journal of Gastroenterology and Hepatology, 25(6), 1029-1041.

24. Dhiman, R. K., \& Chawla, Y. K. (2009). Minimal hepatic encephalopathy. Indian Journal Gastroenterol, 28:5-16.

25. Sharma, P., \& Sharma, B. C. (2010). Predictors of minimal hepatic encephalopathy in patients with cirrhosis. Saudi journal of gastroenterology: official journal of the Saudi Gastroenterology Association, 16(3), 181-187.

26. Bajaj, J. S. (2008). Minimal hepatic encephalopathy matters in daily life. World Journal of Gastroenterology: WJG, 14(23), 36093615.

27. Poordad, F. F. (2007). The burden of hepatic encephalopathy. Alimentary pharmacology \& therapeutics, 25, 3-9.

28. Das, A., Dhiman, R. K., Saraswat, V. A., Verma, M., \& Naik, S. R. (2001). Prevalence and natural history of subclinical hepatic encephalopathy in cirrhosis. Journal of gastroenterology and hepatology, 16(5), 531-535.

29. Romero-Gómez, M., Boza, F., Garcia-Valdecasas, M. S., Garcia, E., \& Aguilar-Reina, J. (2001). Subclinical hepatic encephalopathy predicts the development of overt hepatic encephalopathy. The American journal of gastroenterology,96(9), 2718-2723.

30. Hartmann, I. J., Groeneweg, M., Quero, J. C., Beijeman, S. J., De Man, R. A., Hop, W. C., \& Schalm, S. W. (2000). The prognostic significance of subclinical hepatic encephalopathy. The American journal of gastroenterology,95(8), 2029-2034.

31. Li, Y. Y., Nie, Y. Q., Sha, W. H., Zeng, Z., Yang, F. Y., Ping, L., \& Jia, L. (2004). Prevalence of subclinical hepatic encephalopathy in cirrhotic patients in China. World journal of gastroenterology: WJG, 10(16), 2397-401.

32. Maldonado-Garza, H. J., Vázquez-Elizondo, G., Gaytán-Torres, J. O., Flores-Rendón, Á. R., Cárdenas-Sandoval, M. G., \& Bosques-Padilla, F. J. (2016). Prevalence of minimal hepatic encephalopathy in cirrhotic patients. Annals of hepatology, 10(S2), 40-44.

33. Sterling, R. K., Lissen, E., Clumeck, N., Sola, R., Correa, M. C., Montaner, J., ... \& Messinger, D. (2006). Development of a simple noninvasive index to predict significant fibrosis in patients with HIV/HCV coinfection. Hepatology, 43(6), $1317-$ 1325.

34. Ortiz, M., Jacas, C., \& Córdoba, J. (2005). Minimal hepatic encephalopathy: diagnosis, clinical significance and recommendations. Journal of hepatology, 42(1), S45-S53.

35. Bajaj, J. S., Hafeezullah, M., Hoffmann, R. G., Varma, R. R., Franco, J., Binion, D. G., ... \& Saeian, K. (2008). Navigation skill impairment: another dimension of the driving difficulties in minimal hepatic encephalopathy. Hepatology, 47(2), 596-604.

36. Bajaj, J. S., Hafeezullah, M., Hoffmann, R. G., \& Saeian, K. (2007). Minimal hepatic encephalopathy: a vehicle for accidents and traffic violations. The American journal of gastroenterology, 102(9), 1903-1909.

37. Hartmann, I. J., Groeneweg, M., Quero, J. C., Beijeman, S. J., De Man, R. A., Hop, W. C., \& Schalm, S. W. (2000). The prognostic significance of subclinical hepatic encephalopathy. The American journal of gastroenterology,95(8), 2029-2034.

38. Sidhu, S. S., Goyal, O., Mishra, B. P., Sood, A., Chhina, R. S., \& Soni, R. K. (2011). Rifaximin improves psychometric performance and healthrelated quality of life in patients with minimal hepatic encephalopathy (the RIME Trial). The American journal of gastroenterology, 106(2), 307-316.

39. Kircheis, G., Nilius, R., Held, C., Berndt, H., Buchner, M., Gortelmeyer, R., ... \& Otto, H. (1997). Therapeutic efficacy of LornithineLaspartate infusions in patients with cirrhosis and hepatic encephalopathy: results of a placebocontrolled, study. Hepatology, 25(6), 1351-1360.

40. Dhiman, R. K., Saraswat, V. A., Verma, M., \& Naik, S. R. (1995). Figure connection test: a universal test for assessment of mental state. Journal of gastroenterology and hepatology, 10(1), 14-23. 